\title{
Wannier-Bloch Approach to Localization in High-Harmonics Generation in Solids
}

\author{
Edyta N. Osika, ${ }^{1,2, *}$ Alexis Chacón, ${ }^{2, \dagger}$ Lisa Ortmann, ${ }^{3}$ Noslen Suárez, ${ }^{2}$ Jose Antonio Pérez-Hernández, ${ }^{4}$ \\ Bartłomiej Szafran, ${ }^{1}$ Marcelo F. Ciappina, ${ }^{5,6}$ Fernando Sols, ${ }^{2,7}$ Alexandra S. Landsman, ${ }^{3,8}$ and Maciej Lewenstein ${ }^{2,9}$ \\ ${ }^{1}$ AGH University of Science and Technology, Faculty of Physics and Applied Computer Science, \\ al. Mickiewicza 30, 30-059 Kraków, Poland \\ ${ }^{2}$ ICFO-Institut de Ciencies Fotoniques, The Barcelona Institute of Science and Technology, \\ 08860 Castelldefels (Barcelona), Spain \\ ${ }^{3}$ Max-Planck Institut für Physik komplexer Systeme, Nöthnitzer-Strasse 38, D-01187 Dresden, Germany \\ ${ }^{4}$ Centro de Láseres Pulsados (CLPU), Parque Científico, E-37185 Villamayor, Salamanca, Spain \\ ${ }^{5}$ Max-Planck-Institut für Quantenoptik, Hans-Kopfermann-Strasse 1, 85748 Garching, Germany \\ ${ }^{6}$ Institute of Physics of the ASCR, ELI-Beamlines, Na Slovance 2, 18221 Prague, Czech Republic \\ ${ }^{7}$ Departamento de Física de Materiales, Universidad Complutense de Madrid, E-28040 Madrid, Spain \\ ${ }^{8}$ Department of Physics, Max Planck Postech, Pohang, Gyeongbuk 37673, Republic of Korea \\ ${ }^{9}$ ICREA, Passeig Lluís Companys 23, 08010 Barcelona, Spain
}

(Received 28 July 2016; revised manuscript received 20 January 2017; published 8 May 2017)

Emission of high-order harmonics from solids provides a new avenue in attosecond science. On the one hand, it allows us to investigate fundamental processes of the nonlinear response of electrons driven by a strong laser pulse in a periodic crystal lattice. On the other hand, it opens new paths toward efficient attosecond pulse generation, novel imaging of electronic wave functions, and enhancement of high-order harmonic-generation (HHG) intensity. A key feature of $\mathrm{HHG}$ in a solid (as compared to the wellunderstood phenomenon of HHG in an atomic gas) is the delocalization of the process, whereby an electron ionized from one site in the periodic lattice may recombine in any other. Here, we develop an analytic model, based on the localized Wannier wave functions in the valence band and delocalized Bloch functions in the conduction band. This Wannier-Bloch approach assesses the contributions of individual lattice sites to the HHG process and hence precisely addresses the question of localization of harmonic emission in solids. We apply this model to investigate $\mathrm{HHG}$ in a $\mathrm{ZnO}$ crystal for two different orientations, corresponding to wider and narrower valence and conduction bands, respectively. Interestingly, for narrower bands, the HHG process shows significant localization, similar to harmonic generation in atoms. For all cases, the delocalized contributions to HHG emission are highest near the band-gap energy. Our results pave the way to controlling localized contributions to HHG in a solid crystal.

DOI: 10.1103/PhysRevX.7.021017

\section{INTRODUCTION}

Recently, the techniques of attosecond science, traditionally applied to atoms and molecules in the gas phase [1], have been extended to the solid state [2-12]. A crucial difference between solid and gas targets is the localization of the initial-state electron wave function, which is spatially confined in isolated atoms and molecules but can be delocalized in a solid. The effect of wave-function localization on key aspects of light-solid interaction remains intensely debated. Some attosecond experiments [11,12] on photoemission from metal surfaces suggest that the

\footnotetext{
*edyta.osika@fis.agh.edu.pl

alexis.chacon@icfo.eu
}

Published by the American Physical Society under the terms of the Creative Commons Attribution 4.0 International license. Further distribution of this work must maintain attribution to the author(s) and the published article's title, journal citation, and DOI.
Subject Areas: Condensed Matter Physics, Optics, Quantum Physics

localization of the core-band electrons results in relatively large ionization delays, attributed to transport [13], compared to photoemission from delocalized conduction-band states. Other experiments probing photoemission from the same initial state at different photon energies found that larger ionization delays came from resonant excitation into bulk excited states, rather than from the initial localization of the wave function [14,15].

In this work, we investigate electron localization and the underlying microscopic nonlinear response by focusing on the process of high-harmonic generation (HHG) in a crystal solid. HHG, a cornerstone of attosecond science, has traditionally relied on gas-phase atomic targets and has only recently been demonstrated experimentally in the condensed phase [5-9,16]. A key feature of HHG in atoms is the recombination of the ionized electron with its parent ion, making it a highly localized process. This localization both dramatically limits HHG efficiency and leads to an exponential decline of HHG yield with increasing ellipticity of laser light since ellipticity induces a drift, which 
exponentially suppresses the return of the ionized electron to the parent ion [17].

In contrast, the HHG process in a solid can be delocalized since an electron ionized from one site of the crystal lattice may recombine with any other. However, little is understood about the specifics of this process. For instance, Ghimire et al. [5] found a much weaker dependence of high-harmonic yield on ellipticity in solid $\mathrm{ZnO}$ than would be expected for a gaseous medium, suggesting a highly delocalized process. A significantly stronger ellipticity dependence (although still weaker than in atoms) in the same target was subsequently found in a theoretical work [18], which shows a 2-3 orders of magnitude drop in HHG yield for ellipticity of 0.5 (compared to only a factor of 5 drop measured in Ghimire et al. [5]). At the same time, a recent experiment on solid argon found the same dependence on ellipticity as in gas-phase Ar, suggesting the electron recombines with the same lattice site that it was emitted from [9]. The extent of spatial localization, measured experimentally by ellipticity dependence, is believed to be important for attosecond pulse generation and imaging of the electronic wave functions in the solid state $[9,19]$.

Here, we investigate the spatial dependence of the HHG process in $\mathrm{ZnO}$ by introducing an analytic model, which uses localized Wannier wave functions in the valence band and delocalized Bloch functions in the conduction band. Prior seminal work $[18,20,21]$ used delocalized Bloch functions both in the valence and the conduction bands, thus without prospects to study the spatial resolution of the HHG process. In addition to adequately calculating the total HHG yield, the present Wannier-Bloch approach allows us to separate the contributions of individual lattice sites to each harmonic and hence determine the degree of localization of the HHG process as a function of experimental parameters. We find that this localization varies significantly both with the harmonic order and with the orientation of a crystal. Our results point to the possibility of controlling the spatial localization of the HHG process, with implications for HHG efficiency, imaging of attosecond electron dynamics in condensed matter, and for the emerging area of atto-nanoscience as a whole [22].

\section{WANNIER-BLOCH DESCRIPTION OF HIGH-ORDER HARMONIC GENERATION IN SOLIDS}

Analogous to the three-step model for atoms [23], the HHG in a crystal solid via interband transitions can be described as a sequence of three stages [18]: (i) electron tunneling excitation from the valence band to the conduction one, (ii) electron (hole) acceleration in the conduction (valence) band, and (iii) electron-hole recombination, resulting in an emission of a high harmonic that is a multiple of the frequency of the driving laser.
In most recent experiments, the laser-field strength $\mathcal{E}_{0}$ across the lattice constant $a$ is comparable to the band-gap energy $E_{g}$ of a typical semiconductor $\left(\mathcal{E}_{0} a \simeq E_{g} \simeq\right.$ few eV). As a consequence, the field cannot be considered as a small perturbation [24]. In our model, we therefore assume that this condition is satisfied, but the laser-field amplitude is below the damage threshold. In addition, the photon energy of the laser field should be much smaller than the typical band-gap energy. This means that we restrict our studies to the photon energies in the MIR domain $\left(\hbar \omega_{0} \leq 0.5 \mathrm{eV}\right)$, which implies that the centrallaser wavelength $\lambda_{0}$ is much larger than the typical lattice constant, $a$. Therefore, the dipole approximation is valid for our description of laser-solid interaction. We assume that the laser field is linearly polarized in the $x$ direction, which allows us to adopt a one-dimensional description. The Hamiltonian of a single electron in a crystal under the action of a laser field is given by

$$
H(t)=H_{0}+U_{\text {int }}(x, t),
$$

where

$$
H_{0}=-\frac{1}{2} \frac{\partial^{2}}{\partial x^{2}}+U(x)
$$

is the laser-free Hamiltonian, with $U(x)$ the lattice periodic potential. In Eq. (1), $U_{\text {int }}(x, t)=-q_{e} x E(t)$ is the oscillating potential due to the laser, written in the length gauge. Here, we use atomic units $\hbar=\left|q_{e}\right|=m_{e}=1$, where $q_{e}$ and $m_{e}$ are the electron charge and electron mass, respectively. The laser pulse has the form $E(t)=$ $\mathcal{E}_{0} \sin ^{2}\left(\omega_{0} t / 2 N\right) \sin \left(\omega_{0} t+\varphi_{\text {CEP }}\right)$, where $\mathcal{E}_{0}$ is the electricfield peak amplitude, $\omega_{0}$ the carrier frequency, and $\varphi_{\mathrm{CEP}}$ the carrier-envelope phase (CEP) of the laser field, while $N$ is the number of laser-period cycles.

Unlike the prior work $[18,20]$, we describe the system within a mixed representation: Wannier states in the valence band and Bloch states in the conduction band. In contrast to the Bloch functions, the Wannier functions are spatially localized "elements" of an $L^{2}$ space. In terms of localized wave functions, they thereby provide an analogous insight into the HHG mechanism as the usual approach used in atomic and molecular systems. Furthermore, the Wannier functions form a complete orthogonal set in the valence band but are not eigenfunctions of the Hamiltonian $H_{0}$. In our problem, the initial state corresponds to a completely filled valence band, i.e., a completely filled Fermi sea. This means that initially all the Bloch states are occupied or, equivalently, that all Wannier states are occupied. Thus, we have to solve the time-dependent Schrödinger equation, starting with each Wannier state, and sum up the results at the end. We introduce an ansatz for the complete time-dependent states of a single electron in a lattice as a superposition of 
Wannier states $\left|w_{v, j}\right\rangle$ from the valence band and Bloch states $\left|\phi_{c, k}\right\rangle$ from the conduction band,

$$
|\Psi(t)\rangle=\sum_{j}\left|w_{v, j}\right\rangle a_{j}(t)+\int_{\mathrm{BZ}} a_{c}(k, t)\left|\phi_{c, k}\right\rangle d k,
$$

with the initial condition $a_{j}(0)=\delta_{j, j_{0}}$; i.e., the electron starts the dynamics at the site $j_{0}$. Here, $j$ runs over all atomic sites in the crystal. The Bloch functions of an $m$ th band ( $m=v$ for the valence band and $m=c$ for the conduction band) have the form

$$
\phi_{m, k}(x)=u_{m, k}(x) e^{i k x},
$$

where $u_{m, k}$ is a periodic function with the same periodicity as the crystal. The wave functions in Eq. (4) can be equivalently represented by a set of Wannier functions,

$$
w_{m, j}(x)=\int_{\mathrm{BZ}} \phi_{m, k}\left(x-x_{j}\right) \tilde{w}_{m}(k) d k
$$

where $\tilde{w}_{m}(k)$ is a product of a normalization constant and a phase depending on electron momentum $k$. It has been shown in Ref. [25] that, for a 1D lattice, the $\tilde{w}_{m}$ are independent of $k$, provided the Wannier functions are real and symmetric under appropriate reflection, and that they fall off exponentially with distance. To calculate the emitted harmonics, first we compute the time-dependent dipole moment

$$
\begin{aligned}
d(t) & =-\langle\Psi(t)|x| \Psi(t)\rangle \\
& \approx-\int d x \int_{\mathrm{BZ}} d k \sum_{j} x w_{v, j}^{*}(x) a_{j}^{*}(t) \phi_{c, k}(x) a_{c}(k, t)+\text { c.c. } \\
& =\int_{\mathrm{BZ}} d k \sum_{j} a_{j}^{*}(t) d_{j c}(k) a_{c}(k, t)+\text { c.c. }
\end{aligned}
$$

where $d_{j c}(k)$ is a dipole transition matrix between Wannier $w_{v, j}(k)$ and Bloch $\phi_{c, k}(x)$ states. The physical meaning of this equation can be summarized as follows: At the observed time $t$, the electron previously promoted to the conduction band recombines with the valence band via $d_{j c}(k)$ and emits a photon with an amplitude that depends on the amplitudes $a_{j}(t)$ and $a_{c}(k, t)$. The harmonic emission is then obtained by modulus squared of the Fourier transform of Eq. (6),

$$
\begin{aligned}
I_{\mathrm{HHG}}(\omega) & =\omega^{2}|\tilde{d}(\omega)|^{2} \\
\tilde{d}(\omega) & =\frac{1}{\sqrt{2 \pi}} \int_{-\infty}^{\infty} d t e^{i \omega t} d(t) .
\end{aligned}
$$

According to Vampa et al. [18], at long-laser wavelengths, i.e., between 1.0 and $5.0 \mu \mathrm{m}$, the main contribution to the harmonic spectrum is from interband transitions. Nevertheless, the present model could include the intraband contribution to the dipole moment $d(t)$ by considering the terms $\left\langle w_{j, k}|x| w_{j, k^{\prime}}\right\rangle$ and $\left\langle\phi_{c, k}|x| \phi_{c, k^{\prime}}\right\rangle$ in Eq. (6). We do include the full intraband dynamics in the evolution of $a_{c}(k, t)$ and $a_{j}(t)$ but only to the extent that it influences the interband matrix elements contributing to $d(t)$.

The next step is to compute the dipole transition $d_{j c}(k)$ and the transition amplitudes $a_{c}(k, t)$ and $a_{j}(t)$. The dipole moment $d_{j c}$ can be further expressed as a product of two terms: one dependent and one independent of $j$. First, following Ref. [18], we approximate the matrix elements as follows:

$$
\begin{aligned}
\left\langle\phi_{c, k}|x| \phi_{c, k^{\prime}}\right\rangle & =i \nabla_{k} \delta\left(k-k^{\prime}\right), \\
\left\langle\phi_{c, k}|x| \phi_{v, k^{\prime}}\right\rangle & =-d_{c v}(k) \delta\left(k-k^{\prime}\right),
\end{aligned}
$$

with $d_{c v}(k)=-\left\langle\phi_{c, k}|x| \phi_{v, k}\right\rangle$. The transition dipole moment from the conduction to the valence band is then

$$
\begin{aligned}
d_{j c}(k)= & -\int d x w_{v, j}^{*}(x) x \phi_{c, k}(x) \\
= & -\int d x \int_{\mathrm{BZ}} d k^{\prime} \phi_{v, k^{\prime}}^{*}\left(x-x_{j}\right) \tilde{w}_{v}^{*}\left(x-x_{j}\right) \\
& \times \phi_{c, k}\left(x-x_{j}\right) e^{i k x_{j}} \\
= & d_{v c}(k) \tilde{w}_{v}^{*} e^{i k x_{j}}
\end{aligned}
$$

The replacement of $x$ by $\left(x-x_{j}\right)$ in the above formula is justified by the fact that $\left\langle w_{v, j}\left|\left(x-x_{j}\right)\right| \phi_{c, k}\right\rangle=$ $\left\langle w_{v, j}|x| \phi_{c, k}\right\rangle$. In addition, to obtain the coefficients $a_{j}(t)$ and $a_{c}(k, t)$, we employ the time-dependent Schrödinger equation, Eq. (1), with the wave functions defined in Eq. (3),

$$
i \frac{\partial}{\partial t}|\Psi(t)\rangle=H(t)|\Psi(t)\rangle
$$

For the description of the band structure, we use the tightbinding approximation and assume the dispersion relations for the valence and conduction bands,

$$
\begin{aligned}
& E_{v}(k)=-2 I_{v} \cos (k a), \\
& E_{c}(k)=E_{c}^{\prime}-2 I_{c} \cos (k a),
\end{aligned}
$$

where $I_{v}<0$ and $I_{c}>0$ are hopping parameters in the valence and the conduction bands, respectively, $a$ is a lattice constant, $E_{c}^{\prime}=E_{g}+2 I_{c}-2 I_{v}$, and $E_{g}>0$ is the band-gap energy. The matrix elements for the unperturbed Hamiltonian in both the valence and the conduction bands read

$$
\begin{aligned}
\left\langle w_{v j}\left|H_{0}\right| w_{v j^{\prime}}\right\rangle & =-I_{v} \delta_{\left|j-j^{\prime}\right|, 1}, \\
\left\langle\phi_{c, k}\left|H_{0}\right| \phi_{c, k^{\prime}}\right\rangle & =E_{c}(k) \delta\left(k-k^{\prime}\right),
\end{aligned}
$$


respectively. Therefore, with the previous definitions and after introducing the wave functions Eq. (3) into Eq. (11), we end up with a system of coupled differential equations for $a_{j}(t)$ and $a_{c}(k, t)$,

$$
\begin{gathered}
\dot{a}_{j}(t)=i I_{v} a_{j-1}(t)+i I_{v} a_{j+1}(t)-i x_{j} E(t) a_{j}(t) \\
+i E(t) \int_{\mathrm{BZ}} d k d_{j c}(k) a_{c}(k, t), \\
\dot{a}_{c}(k, t)=-i E_{c}(k) a_{c}(k, t)-E(t) \nabla_{k} a_{c}(k, t) \\
+i E(t) \sum_{j} d_{j c}^{*}(k) a_{j}(t) .
\end{gathered}
$$

Here, we assume only nearest-neighbor hopping in the tight-binding approximation for the valence band (only $a_{j-1}$ and $a_{j+1}$ appear in the formula for $\dot{a}_{j}$ ). In solving Eq. (14), we take into consideration dynamics only due to the hopping in the lattice [the first two terms in Eq. (14)] and the laser electric field (the third term). For weak laser fields, $\mathcal{E}_{0} \sim 10^{-3}$ a.u., one can neglect the last term of Eq. (14), which scales as $\mathcal{E}_{0}^{2}$. This is because the transition amplitude $a_{c}(k, t)$ is proportional to the oscillating field strength $\mathcal{E}_{0}$.

We note from Eq. (15) that the coefficients $a_{c}(k, t)$ are directly related to $a_{j}(t)$, which, in turn, denotes the origin from which the electron will be excited from the valence to the conduction band. This provides a localized viewpoint that is quite distinct from the one of the Bloch-Bloch approach [18]. By neglecting the last term in Eq. (14) and solving it explicitly [26], we obtain

$$
\begin{aligned}
a_{j}(t)= & \sum_{q} a_{q}(0) e^{i q a A(t)}(-\tilde{\lambda})^{j-q} \\
& \times J_{q-j}\left(-2 I_{v}\left[v^{2}(t)+u^{2}(t)\right]^{1 / 2}\right),
\end{aligned}
$$

where $q$ runs over all atomic sites, $a$ is a lattice constant, $J_{q}$ are Bessel functions of $q$ th order, and

$$
\begin{gathered}
A(t)=-\int_{0}^{t} d t^{\prime} E\left(t^{\prime}\right), \\
u(t)=\int_{0}^{t} d t^{\prime} \cos \left[a\left(A(t)-A\left(t^{\prime}\right)\right)\right], \\
v(t)=\int_{0}^{t} d t^{\prime} \sin \left[a\left(A(t)-A\left(t^{\prime}\right)\right)\right], \\
\tilde{\lambda}=\{[v(t)-i u(t)] /[v(t)+i u(t)]\}^{1 / 2} .
\end{gathered}
$$

Here, $A(t)$ is the laser vector potential $E(t)=-\{[\partial A(t)] / \partial t\}$. We assume that the electron is initially localized at one atomic site $j_{0}$, i.e., $a_{q}(0)=\delta_{q j_{0}}$. Later, because of the interatomic hopping and the acceleration driven by the laser electric field, the electron's wave function spreads in the lattice following Eq. (16). The width of the electron's wave-function spread in the lattice at the end of the laser pulse depends on the hopping amplitude $I_{v}$, the lattice constant $a$, the laser-electric-field strength $\mathcal{E}_{0}$, and pulse duration. We allow all coefficients $a_{j}(t)$ to acquire nonzero values during the laser pulse. Equation (15) is solved by following Refs. [18,27], using a change from the quasikinetic momentum reference frame $k$ to the canonical momentum $p=k-A(t)$ defined in the Brillouin zone shifted by $A(t)$, i.e., $\tilde{\mathrm{BZ}}=B Z-A(t)$,

$$
\begin{aligned}
a_{c}(p, t)= & i \sum_{j^{\prime}} \int_{0}^{t} d t^{\prime} E\left(t^{\prime}\right) a_{j^{\prime}}\left(t^{\prime}\right) d_{j c}^{*}\left(p+A\left(t^{\prime}\right)\right) \\
& \times e^{-i \int_{t^{\prime}}^{t} E_{c}\left(p+A\left(t^{\prime \prime}\right)\right) d t^{\prime \prime}} .
\end{aligned}
$$

Note that this canonical momentum $p$ is an invariant of motion for each electron trajectory within $t^{\prime}$ and $t$. Finally, the time-dependent dipole moment $d(t)$ takes the form

$$
\begin{aligned}
d(t)= & i\left|\tilde{w}_{v}\right|^{2} \sum_{j} \sum_{j^{\prime}} \int_{0}^{t} d t^{\prime} \\
& \times \int_{\tilde{\mathrm{BZ}}} d p a_{j}^{*}(t) d_{v c}(p+A(t)) e^{i(p+A(t)) x_{j}} \\
& \times e^{-i \varphi\left(p, t, t^{\prime}\right)} a_{j^{\prime}}\left(t^{\prime}\right) d_{v c}^{*}\left(p+A\left(t^{\prime}\right)\right) e^{-i\left(p+A\left(t^{\prime}\right)\right) x_{j^{\prime}}} E\left(t^{\prime}\right) \\
& + \text { c.c. },
\end{aligned}
$$

where $\varphi\left(p, t, t^{\prime}\right)=\int_{t^{\prime}}^{t} E_{c}\left(p+A\left(t^{\prime \prime}\right)\right) d t^{\prime \prime}$ is the accumulated phase of the electron in the conduction band. Equation (22) describes the harmonic emission originating from a single electron in a lattice. The whole set of localized Wannier functions remains orthogonal during the unitary time evolution. Then, one can expect that the interpretation of the dipole radiation $d(t)$ will be similar to the atomic case. The interpretation of Eq. (22) reads as follows:

(1) The electron located at the $j^{\prime}$ th atomic site is excited from the valence to the conduction band at time $t^{\prime}$ (see Fig. 1).

(2) During the interval of time $\left[t^{\prime}, t\right]$, this excited electron is propagated via $e^{-i \varphi\left(p, t, t^{\prime}\right)}$ and accelerated in the conduction band $E_{c}(p+A(t))$ by means of the laser field. The electron gains energy depending on both the shape of the conduction band dispersion relation and the laser electric field. Note that, for this time interval, the electron wave function spreads along the periodic lattice structure.

(3) Finally, at time $t$, the electron recombines at the $j$ th site (see the arrow pointing down in Fig. 1). As a result, the excess electron energy is emitted in the form of a high-harmonic photon of the fundamental laser frequency. 


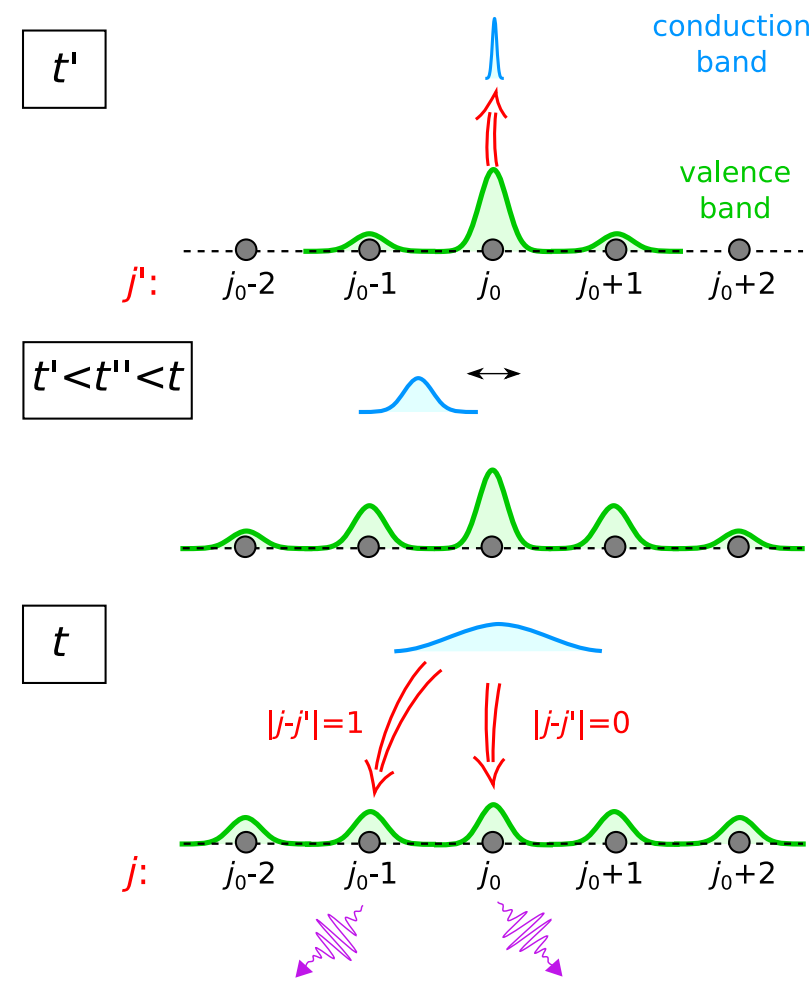

FIG. 1. Scheme of the electron excitation-recombination process for the HHG from the periodic lattice crystal. The periodic lattice is represented by gray disks along the black dashed line. The Wannier wave function is depicted by the green area. At time $t^{\prime}$, a single-electron wave packet is excited from the $j^{\prime}$ th atomic site in the valence band to the conduction band. This wave packet is denoted by blue area. Then, during $\left(t^{\prime}, t\right)$, it is accelerated within the conduction band by the driving laser field. Finally, at time $t$, the electron recombination takes place on different $j$ atomic sites with the subsequent high-harmonic emissions, which are denoted by violet pulse oscillations. Note that the red arrows show excitation at $j^{\prime}=j_{0}$ and recombination at $j=j_{0}$ and $j_{0}-1$ atomic sites.

This picture is based on the stationary phase approximation applied to rapidly oscillating integrands. We apply this method to the Fourier transform of the dipole moment $d(t)$ and discuss it in Appendix A for the integral with respect to the excitation time $t^{\prime}$ and emission time $t$. The excitation mechanism at time $t^{\prime}$ can be viewed as tunneling because $t^{\prime}$ is complex $[18,27,28]$. In addition, the saddlepoint conditions at the recombination time $t$ show that the photon energy emitted by solids is determined by the conduction-band energy-relation dispersion, the relative excitation-recombination position, and the phase of the coefficient $a_{j}(t)$.

Via the semiclassical saddle-point analysis of the momentum integral in Eq. (22) (see Appendix A), one can identify the relative electron trajectory contributions or, more precisely, relative excitation-recombination site contributions to the HHG spectrum. This suggests that there is a set of electron trajectories (long and short) that are born at site $j^{\prime}$ and recombine at site $j$. In particular, the complex double sum over $j^{\prime}$ and $j$ of the dipole radiation $d(t)$ contains all the relative excitation-recombination site contributions to the HHG process. Therefore, the harmonic emission is coherent as long as dephasing is neglected.

The dephasing effect, while noticeable, does not change the general form of the harmonic spectrum of $\mathrm{ZnO}$, as previously found in Ref. [18]. Therefore, as the main focus is on the basic mechanism behind HHG emission, we neglect the dephasing in the present analysis. The electron accumulates two different "intrinsic" phases during the evolution: (i) the arbitrary phase of the electron wave function, which is constant in time, and (ii) the phase related to the initial position of an electron, which is encoded in the coefficients $a_{j}^{\prime}(t)$ and $a_{j}(t)$ in $\exp [i q a A(t)]$.

Concerning (i), because the Hamiltonian evolution is unitary, this phase cancels out at the recombinationemission time $t$ when the electron recombines with the same wave function that it was excited from.

Phase (ii) plays a role in generalizing this approach from the single-electron picture to the full valence band (all Wannier functions occupied). To account for contributions of all electrons in a lattice, we multiply the dipole moment given by Eq. (22) by the total number of electrons, $N_{e}$. This is justified by cancellation of the two phases arising from shifting the initial Wannier function to another atomic site. One of these phases is related to $a_{j}(t)$ [see Eq. (16)], and the other one comes from the dipole transition $d_{v c}(p+A(t)) e^{i(p+A(t)) x_{j}}$. In particular, let us consider another electron localized at a site $j_{p}=j_{0}+n$ at time $t=0$. In the dipole, Eq. (22), for any given $t, t^{\prime}$ two additional phases will appear in comparison to the calculations where an electron starting at the $j_{0}$ site is used: (i) $\exp \left[-i\left(A\left(t^{\prime}\right)-A(t)\right) n a\right]$ from the shift of $x_{j}, x_{j^{\prime}}$ in Eq. (22), and (ii) $\exp [-i A(t) n a] \exp \left[i A\left(t^{\prime}\right) n a\right]=$ $\exp \left[-i\left(A(t)-A\left(t^{\prime}\right)\right) n a\right]$ from the shift $j \rightarrow j+n, q \rightarrow$ $q+n$ in $a_{j}^{*}(t) a_{j}^{\prime}\left(t^{\prime}\right)$. Since these two phases cancel each other, the contributions from electrons born at all crystal sites to the harmonic emission will be the same.

\section{RESULTS AND DISCUSSION}

Here, we use the model developed in Sec. II to investigate harmonic emission of $\mathrm{ZnO}$. The approach presented in this paper treats the HHG process within an atomisticlike approximation, assuming initial localization of an electron at one atomic site and gradual spread of its wave function in the lattice due to interatomic hopping and the influence of a laser electric field. We consider two different directions for the laser-field polarization, $\Gamma-A$ and $\Gamma-M$ [29]. In the case of a narrow valence band (small values of $I_{v}$ ), the dynamics of the valence electrons is slow, and the electron wave function does not spread much when the laser pulse is turned on. This means that only a few of the $a_{j}$ coefficients in the sum of Eq. (22) will have nonzero values. 
For calculations in Sec. III. B, where we assume laser polarization in the $\Gamma-A$ direction, we consider 15 atomic sites (ions) on both sides of $j_{0}$ (with this number, we already obtain good convergence). In the case of a wide valence band (Sec. III A), where the laser polarization is in the $\Gamma-M$ direction, the dynamics are much faster, and we need to consider up to 1000 atomic sites to obtain convergence.

\section{A. Comparison of Bloch-Bloch and Wannier-Bloch models}

We begin by comparing the emitted HHG spectrum from the Wannier-Bloch approach, Eq. (22), with the spectrum obtained from the delocalized Bloch-Bloch approach. The Bloch-Bloch model was implemented following Ref. [18], with the cosine band structure approximated by a Taylor expansion up to the fourth order and integration in momentum space replaced by a saddle-point approximation. Integration over the ionization time was done numerically using a Gaussian quadrature routine, and the Fourier integral was performed as a fast Fourier Transform (FFT).

Figure 2(a) depicts the HHG spectrum computed using the same laser parameters as in Ref. [20], i.e., laser peak intensity $I_{0}=3.15 \times 10^{11} \mathrm{~W} / \mathrm{cm}^{2}$, carrier wavelength $\lambda_{0}=3.25 \mu \mathrm{m}$, and pulse length of 10 laser periods (FWHM $\sim 53 \mathrm{fs}$ ). For computational convenience, we use a sine-squared envelope laser pulse (defined in Sec. II) instead of the Gaussian one used in Ref. [20]. A simplified cosinelike band structure in the $\Gamma-M$ direction, with the approximate parameters of Ref. [20], is used, i.e., $E_{g}=0.1213$ a.u., $I_{c}=0.0449$ a.u., $I_{v}=-0.0464$ a.u., and lattice constant $a=5.32$ a.u. Following Ref. [18], the dipole moment is assumed to be constant: $d_{v c}(k)=$ 3.46 a.u. For details about the 1D-Bloch-Bloch calculation of Vampa et al., we refer to the Supplemental Material of Ref. [18] and the subsequent article Ref. [20].

Our approach exhibits good agreement with the BlochBloch model, reproducing the plateau, the cutoff, and the standard odd harmonic structure around the cutoff. The two spectra differ mainly in the low-order harmonics region, suggesting that localization (or recombination with the parent atom) may play greater importance in the production of low-order harmonics, as is confirmed in Fig. 2(b). Overall, this comparison confirms that the WannierBloch picture reproduces the essential features of the Bloch-Bloch model for the emitted harmonics.

Figure 2(b) shows the contributions to the harmonic spectra obtained from different components of the $j, j^{\prime}$ sums in Eq. (22). The thick black line indicates the whole harmonic spectrum, and the colored lines show individual contributions corresponding to different distances, $\Delta j=\left|j-j^{\prime}\right|$, between the electron excitation (at $j^{\prime}$ ) and recombination (at $j$ ) atomic sites. To calculate the contribution of a given $\Delta j$, we apply FFT on the part of the
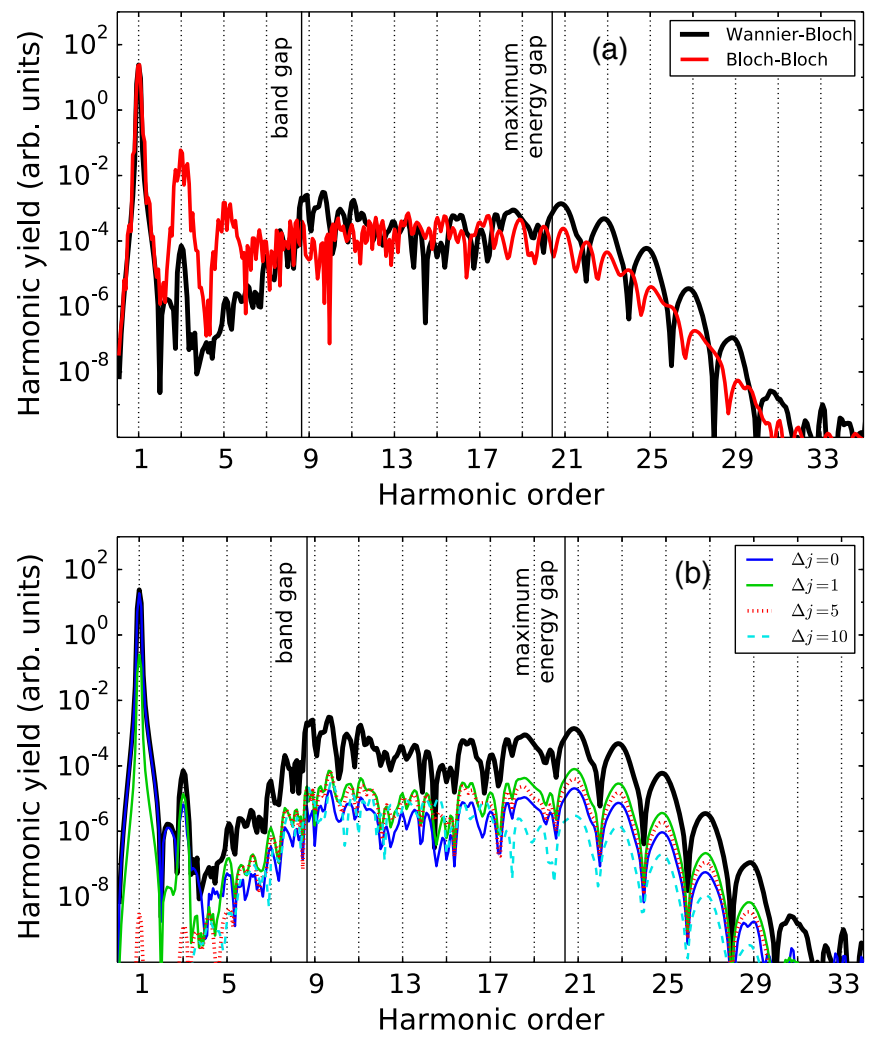

FIG. 2. Harmonic spectra comparison of the Wannier-Bloch approach (black line) with the Bloch-Bloch approach (red line) is shown in panel (a). Decomposition of the harmonic contribution into different lengths of electron-recombination atomic sites $\Delta j$ using the Wannier-Bloch method is depicted in panel (b). The calculations are carried out for laser polarization in the $\Gamma-M$ direction of $\mathrm{ZnO}$ crystal. The laser parameters are as follows: carrier wavelength of $\lambda_{0}=3.25 \mu \mathrm{m}$, laser intensity $I_{0}=3.15 \times 10^{11} \mathrm{~W} / \mathrm{cm}^{2}$, total number of laser cycles $N=10$ periods, and $\varphi_{\mathrm{CEP}}=0$.

dipole $d(t)$ composed only of terms in Eq. (22) for which $\left|j-j^{\prime}\right|$ is equal to $\Delta j$. The relative length of displacement between the excitation and recombination sites is given by $\mathcal{D}_{s}=\Delta j a$, which quantifies the concept of relative excitation-recombination places.

As Fig. 2(b) shows, in certain parts of the spectrum, even $\Delta j=10$ paths contribute considerably to the total harmonic emission. This is in clear contrast to HHG in atomic gas, where the electron has to recombine with its parent atom, corresponding to $\Delta j=0$ contributions only. In the next section, we attempt to understand why even relatively distant atomic sites can contribute significantly to the total emission spectrum in solids.

\section{B. Wannier-Bloch picture}

In order to further investigate the contribution of different relative excitation-recombination places $\mathcal{D}_{s}$ to the $\mathrm{HHG}$ spectrum, we calculate harmonic emission for a set of different laser parameters. This also allows us to establish 
under what conditions the Wannier-Bloch approach may be a more adequate description relative to the Bloch-Bloch one [18]. Because of computational constraints in the HHG spectra calculations using the band structure of Sec. III A, here we focus on the narrower valence band case. For the latter, we are able to scan a wider range of parameters and analyze the band-structure influence on the different relative excitation-recombination contribution sites to the HHG.

In order to compute the HHG spectra, we fix the optical axis of $\mathrm{ZnO}$ (with polarization of the laser in the $\Gamma-A$ direction) [18] and use $a=9.83$ a.u., $I_{c}=0.02175$ a.u., $\quad I_{v}=-0.00295$ a.u., and $E_{g}=$ 0.1213 a.u. Also, following the formulation in Ref. [18], $d_{v c}(k)=\sqrt{\left\{E_{p} /\left[2\left(E_{c}(k)-E_{v}(k)\right)^{2}\right]\right\}}$, with the Kane parameter $E_{p}$ set to 0.479 . Figures 3(a) and 3(b) show the results of the emitted harmonic spectra for two different
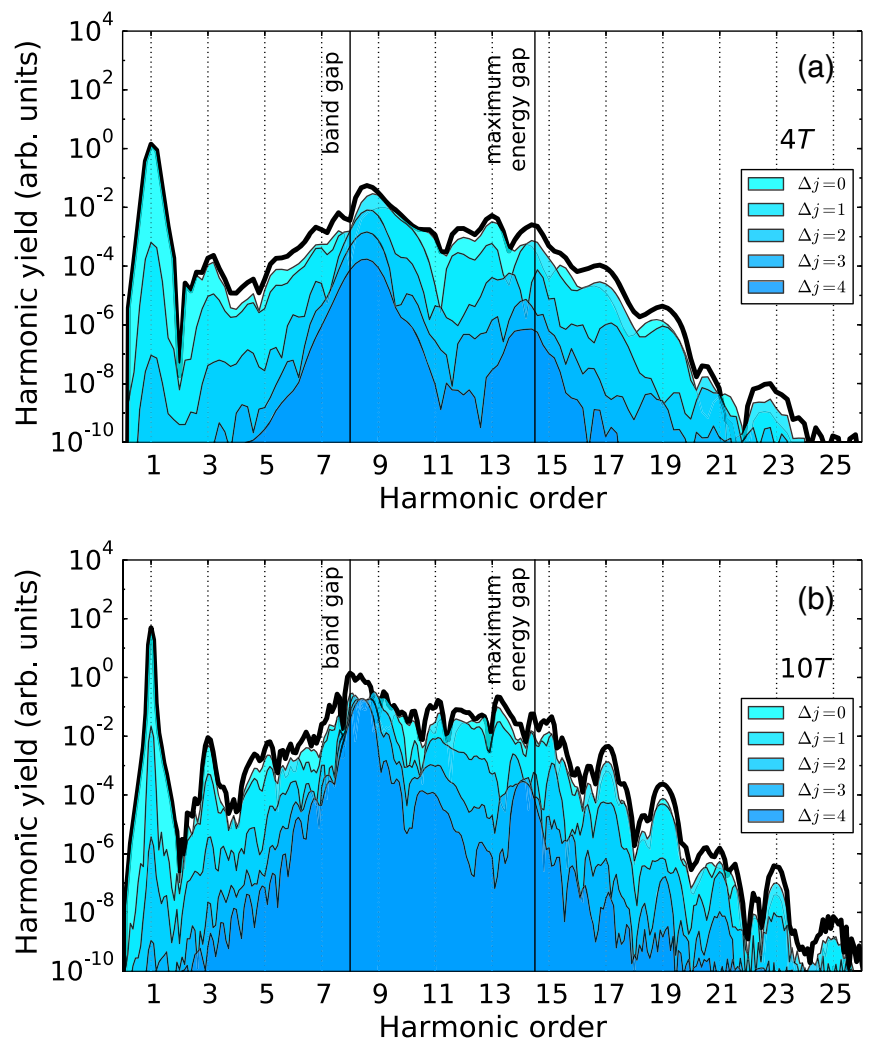

FIG. 3. Panels (a) and (b) depict the full harmonic spectra for two different laser time durations, $N=4$ and 10 , respectively (thick black lines), calculated within the Wannier-Bloch approach. Furthermore, color regions show the harmonic contributions of the different relative electron-recombination atomic sites in the spatially periodic lattice structure $\Delta j=0,1, \ldots, 4$. The black vertical lines (read from left to right) point out the band-gap harmonic and the expected cutoff harmonic orders, respectively. The laser-pulse peak intensity is $I_{0}=5 \times 10^{11} \mathrm{~W} / \mathrm{cm}^{2}$, the carrier wavelength $\lambda_{0}=3.0 \mu \mathrm{m}$, and its CEP is $\varphi_{\mathrm{CEP}}=0 \mathrm{rad}$. The calculations are carried out for laser polarization in the $\Gamma-\mathrm{A}$ direction. laser-pulse durations, namely, $\Delta t_{b}=N T_{0}$, where $N=4$ and $N=10$ are the number of cycles, respectively, and $T_{0}$ the period of the laser field. We observe that the highest contribution to the HHG spectrum comes from $\Delta j=0$, i.e., for the case when the electron recombines to the same atomic-parent site from where it was previously excited to the conduction band. The longer the electronrecombination displacement $\mathcal{D}_{s}$ is, the lower its contribution to the HHG spectra. Both panels of Fig. 3 show that, while, in general, the obtained harmonic yield decays very fast with $\Delta j$, there is a relatively large signal between the 8th and 15th harmonic which is also preserved for larger values of $\Delta j$. This signal corresponds to the energy gap between valence and conduction bands, which spreads from about $8 \omega_{0}$ (band gap for $k=0$ ) to $14.5 \omega_{0}$ (maximum energy gap for $k=(\pi / a)$ ). The greater contribution from large $\Delta j$ processes near the maximum and minimum energy gaps can be understood as resulting from the high density of possible interband transitions involving opposite band edges. Those transitions occur between states with narrowly defined momenta (near the band extrema), which require broad spatial coherence as reflected in large recombination lengths.

Figure 3 shows the typical features of HHG spectra: Namely, odd harmonics are present, the signal is strongest for the low-order harmonics (1st and 3rd), and a plateau region and a cutoff can be easily distinguished. As would be expected for interband emission, the cutoff is located near the harmonic equivalent of the maximum energy difference between the conduction and valence bands. The region of the plateau exhibits pronounced interference structures-there is no clear recognition of even-odd harmonic symmetry. This behavior is also typical of the harmonic spectra in atoms in the limit of short pulses [30,31].

The spectra in Fig. 3 can be compared with the experimental results shown in Fig. 3 of Ref. [5], specifically for the crystal angle $0^{\circ}$. In this experimental data, up to the 13th harmonic is distinguishable, which is close to the cutoff value obtained in our calculations. As predicted by our model, a signal near the band-gap energy is observed in Ref. [5] (where, however, it is attributed to fluorescence effects). In contrast to our results, in the experiment, both odd and even harmonics appear in the spectrum. This is likely an effect of a symmetry breaking in the 3D- $\mathrm{ZnO}$ lattice, which we do not take into account here.

Note that the saddle-point method was not used to solve the momentum integral in Eq. (22). We perform that momentum integral numerically. The argument is based on the fact that the dipole-radiation phase takes into account electron propagation in the conduction band. The latter means that, for narrow bands, the electron cannot accumulate large energy. Therefore, the stationary phase method might be inaccurate in solving that type of momentum integral. Nevertheless, the saddle-point method 
is still suitable for calculating the momentum integral in Eq. (22) for larger-energy band structure.

To investigate how harmonic emission scales as a function of wavelength, we calculate the harmonic spectrum for different values of the laser wavelength, $\lambda_{0}$. The results are shown in Fig. 4. Figure 4(a) shows HHG spectra for wavelengths in a range of $800-3500 \mathrm{~nm}$. The frequency axis for each wavelength is scaled in $\omega_{0}=2 \pi c / \lambda_{0}$ units. It is observed that the cutoff moves to lower harmonics while the wavelength decreases. However, one may expect that the cutoff stays constant in terms of photon energy because of a well-defined maximum energy difference between the conduction and valence bands of about $6 \mathrm{eV}$. This effect is shown in Fig. 4(b), where the spectra of Fig. 4(a) are replotted as a function of photon energy. Hence, the cutoff

(a)
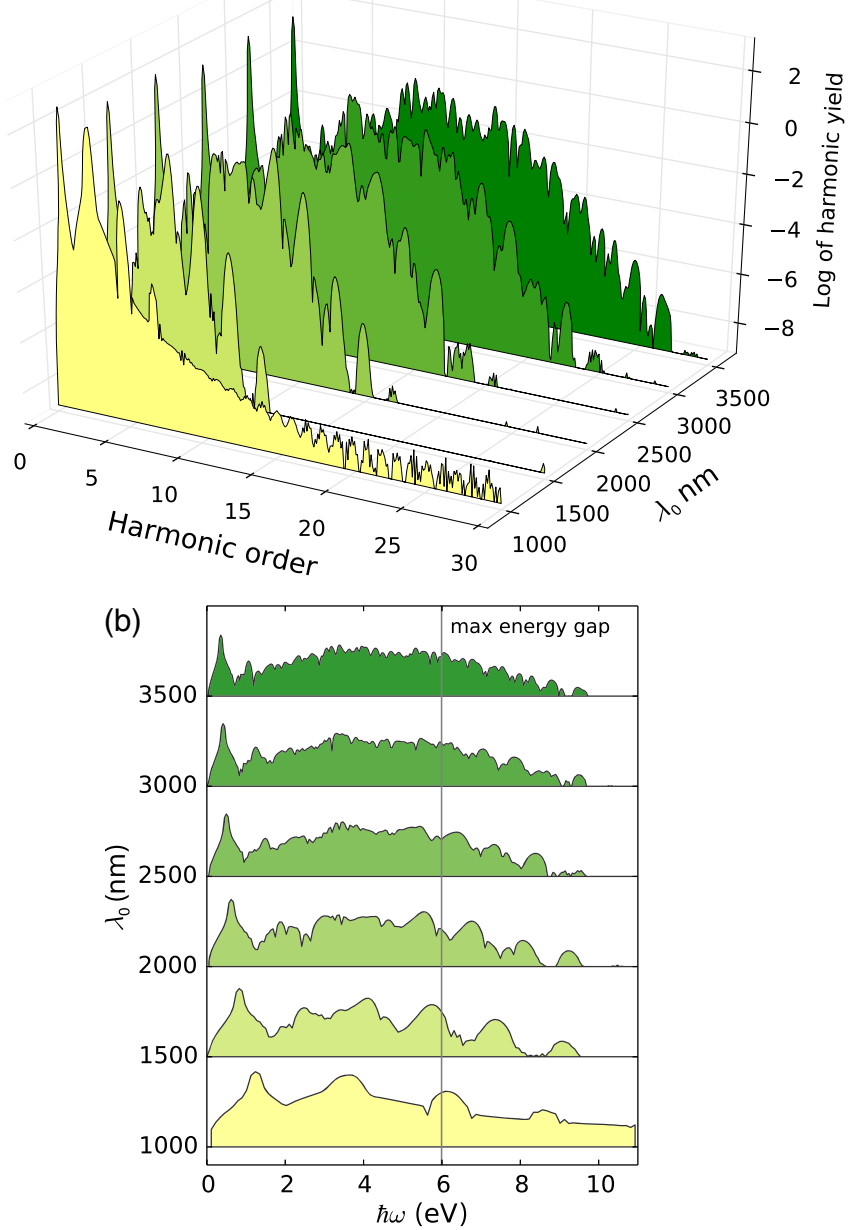

FIG. 4. (a) Harmonic spectra for different values of the carrierlaser wavelength $\lambda_{0}$. (b) Same as (a) but with energy units instead of harmonic order on the frequency axis. The grey line shows maximum emitted photon energy, which corresponds exactly to the maximum energy difference between the conduction and valence bands, $\Delta E_{v c}=6 \mathrm{eV}$. Laser intensity was set to $I_{0}=5 \times 10^{11} \mathrm{~W} / \mathrm{cm}^{2}$, laser-pulse length to 10 laser periods, and $\varphi_{\mathrm{CEP}}=0 \mathrm{rad}$. of the harmonic spectrum is in good agreement with the value calculated from the band structure. From the socalled action phase in Eq. (22), i.e., $\varphi\left(p, t, t^{\prime}\right)$, we can infer that the maximum harmonic energy produced in a solid lattice should be limited by the band dispersion relation (this result is consistent with prior findings [18]).

Previously, it was found that HHG in solids scales linearly with the electric-field strength [5]. Here, we calculate HHG spectra for different values of the laser electric field. Figure 5 shows the HHG spectrum for electric-field amplitudes, in the range $0.4-1.0 \mathcal{E}_{0}$, where $\mathcal{E}_{0}=3.779 \times 10^{-3}$ a.u. corresponds to laser intensity $I_{0}=5 \times 10^{11} \mathrm{~W} / \mathrm{cm}^{2}$. These calculations were performed for the case of a narrow valence band, corresponding to laser polarization along the $\Gamma-\mathrm{A}$ direction. As expected, decreasing the laser intensity shifts the cutoff to lower-order harmonics. In Fig. 5, we trace an estimated straight line over the harmonic-electric field map.

For cases shown in Figs. 4 and 5, the relative contribution of longer displacements, $\mathcal{D}_{s}$, to harmonic emission is similar to what was observed in Fig. 3. In particular, long relative displacements contribute significantly only to the harmonics close to the band-gap energy. Neither change of wavelength nor of laser intensity had a significant effect on the observed tendency. The situation is different for wider band solids, as discussed in Sec. III A.

The linear harmonic cutoff as a function of the electricfield strength was demonstrated experimentally in Ref. [5]. In an attempt to reproduce this experimental finding with the Wannier-Bloch model, we also calculate the cutoff

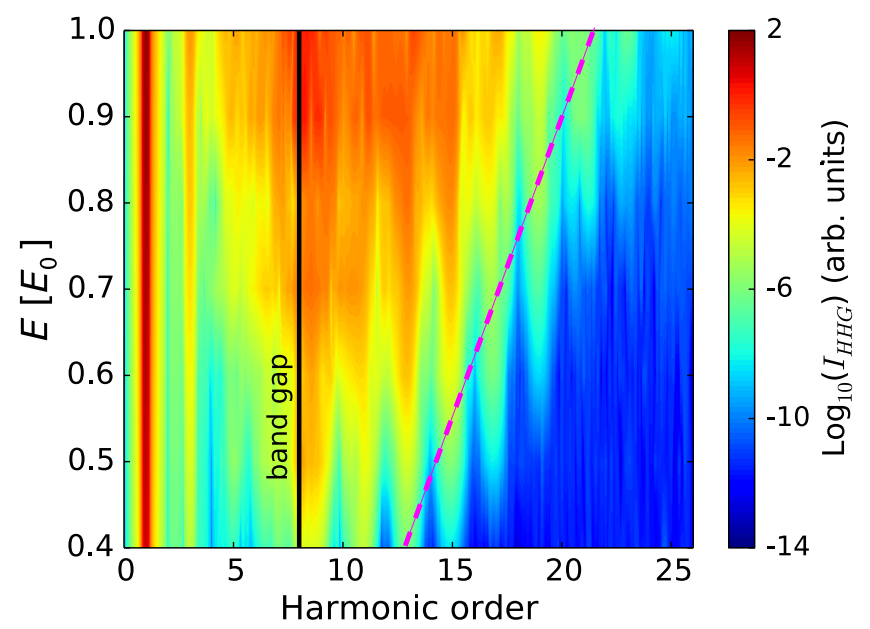

FIG. 5. Harmonic spectra as a function of electric-field strength. The electric-field axis is in units of $\mathcal{E}_{0}=0.0038$ a.u., corresponding to the laser intensity $I_{0}=5 \times 10^{11} \mathrm{~W} / \mathrm{cm}^{2}$. The dashed magenta straight line denotes the estimated cutoff of the harmonic spectrum as a function of the electric-field strength. The carrier-laser wavelength was set to $\lambda_{0}=3.0 \mu \mathrm{m}$, laser-pulse length to $N=10$ laser periods $T_{0}$, and $\varphi_{\text {CEP }}=0 \mathrm{rad}$. The calculations are carried out for laser polarization in the $\Gamma-\mathrm{A}$ direction of $\mathrm{ZnO}$ crystal. 

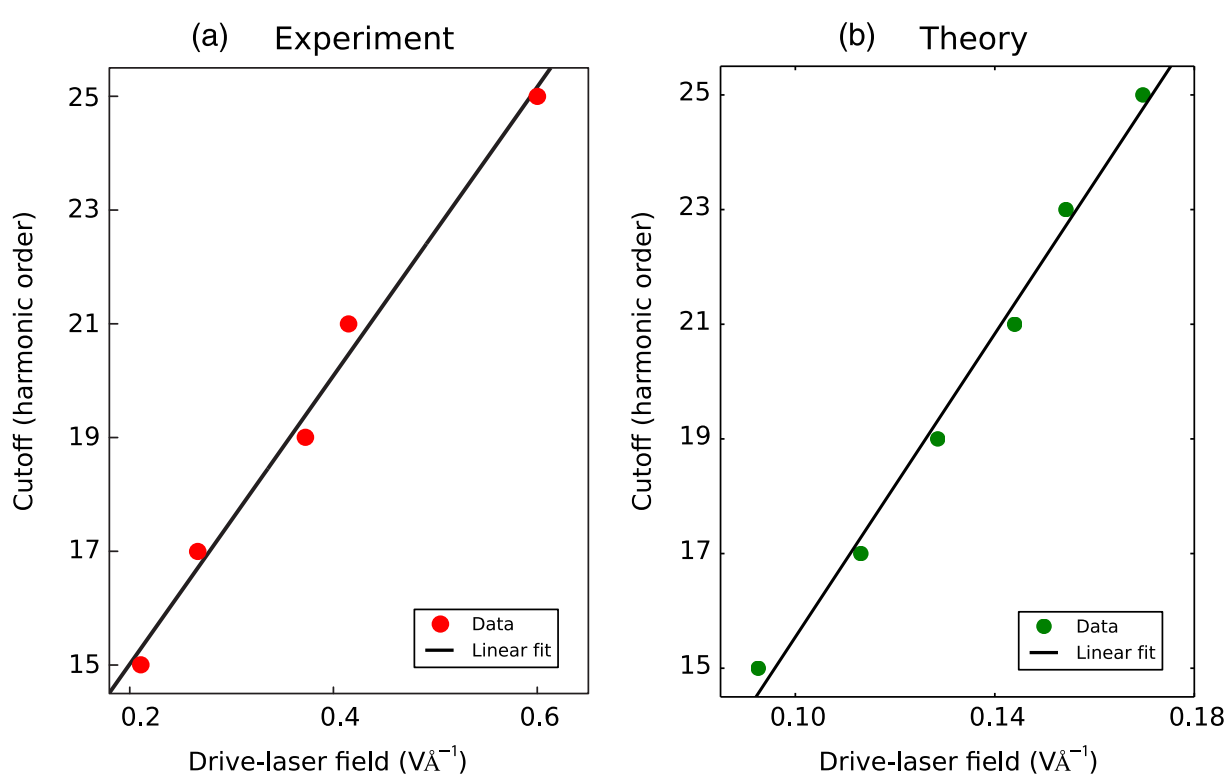

FIG. 6. Experimental and theoretical comparison: Linear scaling of the cutoff harmonic order with the laser electric-field strength as obtained in (a) the experiment [courtesy of Louis DiMauro from Fig. 1(b) of Ref. [5] and (b) theoretical calculations by means of the Wannier-Bloch approach.

dependence on the electric-field strength for laser polarization in the $\Gamma-\mathrm{M}$ direction of $\mathrm{ZnO}$ crystal (wide bands). The comparison of the experimental and theoretical results is presented in Fig. 6. Our results reproduce the linear scaling observed in Ref. [5] quite well. However, our model is rather simplified for analytic tractability: (i) We treat the 1D case; i.e., this underestimates the effects of wave-packet spreading (because there are fewer nearest-neighbor sites), making HHG emission effectively more efficient at lower intensities. (ii) We consider only the single conduction band in the dispersion relation, and we do not include correlation effects or the full 3D band structure of the crystal lattice (iii) We do not include possible (many-body) screening effects that decrease the effective field in the solid and thus make the field necessary for efficient HHG emission larger. (iv) Our Wannier-Bloch theory should, strictly speaking, work best for narrow valence bandshere, it is applied to the case of a moderately broad band, where Wannier wave-function spreading is more significant and more difficult to handle. Nevertheless, close agreement of the cutoff with the Bloch-Bloch approach published in Ref. [18] suggests that our approach can handle these wider bands as well.

Finally, only interband contributions are considered here, complementing the theory discussed by Ghimire et al. [5], which focuses on intraband Bloch oscillations, calculating the resulting oscillating current. Their approach allows one to immediately conclude that the cutoff law is linear in the electric field. However, direct comparison with experimental data is also difficult here, because of, for instance, large sensitivity of the spectrum to the details of the dispersion relation in the conduction band.
We expect that the full treatment of both contributions, and inclusion of the effects discussed above, is necessary to recover close agreement with experimental data. This treatment goes beyond the study of the present paper, which, in the first place, presents a systematic method and, in the second place, discusses results obtained under given approximations, which qualitatively hold in many situations of interest.

Our results agree in many aspects with prior experimental and theoretical observations $[5,8,18,20]$. In particular, (i) the HHG cutoff shows a dependence on the maximum energy difference between the valence and conduction bands, as well as on the laser wavelength and peak intensity; (ii) for long laser pulses and few-cycle laser fields, the model depicts the full odd spectrum and a continuum spectrum, respectively; and (iii) we find a direct link between the emitted harmonic spectrum shape and the band structure. The excellent agreement between our approach and the Bloch-Bloch approach, shown in Fig. 2(a), over harmonics 8-20 suggests that the Wannier-Bloch model could be applied to the reconstruction of the band structure following the approach in Ref. [32].

The delocalization of the process in the $\Gamma-\mathrm{M}$ direction, where even $\Delta j=10$ sites contribute significantly to the HHG yield [see Fig. 2(b)], is confirmed by the experimental results in Ref. [5]. In particular, Ghimire et al. [5] measure a relatively small decline in the HHG yield for ellipticity of 0.5 (see Fig. 4 in Ref. [5]). A much more extreme drop would be found in atomic HHG, which is already suppressed at an ellipticity of 0.2 [17,33-35].

Furthermore, we have performed a classical estimation of the electron trajectories as a function of ellipticity, as 
illustrated in Appendix C. We observe that for nonzero ellipticities, the electron trajectories might recollide with "transverse neighbors" in the periodic lattice structure. Since, quantum mechanically, the electron wave function can spread in a 2D lattice, one can expect that the harmonic emission in solids decreases less than in the gaseous phase.

\section{CONCLUDING REMARKS}

By using localized atomic sites in the valence band and delocalized functions in the conduction band, our model has the closest parallels to harmonic generation from atomic gas. As such, it allows one to access contributions of individual lattice sites and hence assess the degree of localization of HHG in solid-something that has previously been inaccessible. In particular, we can describe a process in which an electron initially localized at the $j^{\prime}$ th atom in the valence band has a certain probability to be excited to the conduction band, where it is accelerated to a high energy before recombining either to the parent atom, at the $j^{\prime}$ th site, or (with different probability) to any other $j$ th atom in the lattice.

Different displacements of the electron-recombination atomic sites, i.e., $\Delta j=\left|j-j^{\prime}\right|$, give different contributions to the harmonic spectrum. The approach developed here allows us to extract all of these contributions. In particular, the main contribution was found to be given by $\Delta j=0$ or by electron recombining at the same atomic site it was excited from. Especially for the case of narrow bands in the band structure, lower $\Delta j$ contribute by far the most to the harmonic spectrum, signifying substantial localization in the HHG process. On the other hand, we found an enhanced contribution of high $\Delta j$ in the case of wider valence and conduction bands. This enhanced delocalization can be viewed as resulting from the small effective mass that the electrons have in a lattice when they populate a broad band with correspondingly high hopping rates. In all cases, distant neighbor contributions were highest near the band-gap energy. This suggests that the harmonic yield near the band-gap energy should decline less (relative to other harmonics) with increasing ellipticity of laser light since elliptical polarization tends to suppress local contributions.

Note that our results, by means of the Wannier-Bloch approach, employ a different framework than those presented by the Bloch-Bloch model in Ref. [18]. While our model recreates the conventional atomic picture, the Bloch-Bloch model is based on the electron-hole pair recombination. Therefore, it is clear that both approaches, while predicting similar total HHG spectra, provide different insights into the physics of the HHG process.

The present approach is particularly suitable to model HHG from nanostructures such as those achieved in the recent paper by Han et al. [36] and those planned in future experiments. We expect the effects of localization to be crucial for such experiments, considering the plasmonic hot spots and the spatial variation of the electric field on a nanometer scale. Hence, a proper understanding of the degree of HHG localization in condensed-matter experiments will be particularly relevant to nanostructures whose geometry may even be designed to suppress or enhance farneighbor contributions. In addition to affecting $\mathrm{HHG}$ efficiency and attosecond pulse generation, suppressing delocalized contributions will also have a big impact on electronic wave-function imaging, as recently pointed out in Ref. [9].

Our results suggest that it should be possible to control the localization of the HHG process by varying experimental parameters. Hence, by quantifying site-specific contributions, our work paves the way to controlling HHG efficiency and imaging of the electronic wave function in a crystal lattice $[9,19]$.

\section{ACKNOWLEDGMENTS}

We are very thankful to Professor Louis DiMauro's group for providing experimental data of Ref. [5]. E. N. O. acknowledges support from the doctoral stipend ETIUDA of the National Science Centre according to Decision No. DEC-2015/16/T/ST3/00266. A. C. and M. L. acknowledge MINECO (National Plan Grant: FISICATEAMO No. FIS2016-79508-P, SEVERO OCHOA No. SEV-20150522, FOQUS No. FIS2013-46768-P), Fundació Cellex Generalitat de Catalunya (AGAUR Grant No. 2014 SGR874 and CERCA/Program), ERC AdG OSYRIS, EU FETPRO QUIC, EU STREP EquaM (FP7/2007-2013 Grant No. 323714) and the National Science Centre, PolandSymfonia Grant No. 2016/20/W/ST4/00314. We also acknowledge support from the European Union's Horizon 2020 research and innovation program under Grant Agreement No. 654148 Laserlab-Europe and Spain's MINECO (Grants No. FURIAM FIS2013-47741-R and No. DYNAMOLS FIS2013-41716-P). We also acknowledge support from PALMA project FIS2016-81056-R. This work was supported by the project ELI-Extreme Light Infrastructure-phase 2 (CZ.02.1.01/0.0/0.0/15_008/ 0000162). A. L. acknowledges partial support by Max Planck Postech/Korea Research Initiative Program (Grant No. 2011-0031558) and the Max Planck Center for Attosecond Science (MPC-AS).

\section{APPENDIX A: SADDLE-POINT APPROXIMATION}

In order to extract physical information about our dipole radiation $d(t)$ by means of the Wannier-Bloch approach, in the following, we employ the saddle-point approximation to the Fourier transform of Eq. (22). This stationary phase method is justified in the case when the accumulated electron energy is large, and thus, the integrand phases rapidly vary with respect to the integration variables.

To compute the harmonic spectrum, the Fourier transform of the dipole radiation $d(t)$ is required, and it reads 


$$
\begin{aligned}
\tilde{d}(\omega)= & i\left|\tilde{w}_{v}\right|^{2} \sum_{j} \sum_{j^{\prime}} \int_{-\infty}^{+\infty} d t e^{i \omega t} \int_{0}^{t} d t^{\prime} \int_{\mathrm{BZ}} d p a_{j}^{*}(t) d_{v c}(p+A(t)) e^{i(p+A(t)) x_{j}} \\
& \times e^{-i \varphi\left(p, t, t^{\prime}\right)} a_{j^{\prime}}\left(t^{\prime}\right) d_{v c}^{*}\left(p+A\left(t^{\prime}\right)\right) e^{-i\left(p+A\left(t^{\prime}\right)\right) x_{j^{\prime}}} E\left(t^{\prime}\right) .
\end{aligned}
$$

Mathematically, one can rewrite the latter equation as a "product" of a global amplitude and phase:

$$
\tilde{d}(\omega)=\sum_{j} \sum_{j^{\prime}} \int_{-\infty}^{+\infty} d t \int_{0}^{t} d t^{\prime} \int_{\tilde{\mathrm{BZ}}} d p f_{j, j^{\prime}}\left(p, t, t^{\prime}\right) e^{-i \Phi_{j, j^{\prime}}\left(p, t, t^{\prime}\right)+i \omega t}
$$

Here, the amplitude $f_{j, j^{\prime}}$ and the phase $\Phi_{j, j^{\prime}}$ are defined according to

$$
\begin{aligned}
f_{j, j^{\prime}}\left(p, t, t^{\prime}\right) & =i\left|\tilde{w}_{v}\right|^{2}\left|a_{j}(t)\right| d_{v c}(p+A(t))\left|a_{j^{\prime}}\left(t^{\prime}\right)\right| d_{v c}^{*}\left(p+A\left(t^{\prime}\right)\right) E\left(t^{\prime}\right), \\
\Phi_{j, j^{\prime}}\left(p, t, t^{\prime}\right) & =\varphi\left(p, t, t^{\prime}\right)-\varphi_{a_{j}}(t)-(p+A(t)) x_{j}+\varphi_{a_{j^{\prime}}}\left(t^{\prime}\right)+\left(p+A\left(t^{\prime}\right)\right) x_{j^{\prime}},
\end{aligned}
$$

where $\varphi\left(p, t, t^{\prime}\right)$ is the electron accumulation phase in the conduction band. Note that $\varphi_{\left.a_{j^{\prime}} t^{\prime}\right)}\left[\varphi_{a_{j}}(t)\right]$ is the phase associated with the population coefficient of the whole wave function $a_{j^{\prime}}\left(t^{\prime}\right)\left[a_{j}(t)\right]$ in the valence band at the corresponding excitation time $t^{\prime}$ (emission $t$ ) and site $j^{\prime}$ (emission site $j$ ).

The high-order harmonic generation is a highly nonlinear process. Then, one can expect the phases $\Phi_{j, j^{\prime}}\left(p, t, t^{\prime}\right)$ and the "full phase" $\tilde{\Phi}\left(p, t, t^{\prime}\right)=\Phi_{j, j^{\prime}}\left(p, t, t^{\prime}\right)-\omega t$, in the respective integrals on the excitation time $t^{\prime}$ and the emission time $t$, quickly vary. This justifies a semiclassical analysis by means of the saddle-point approximation $[18,27,28]$. In order to compute the saddle points, times $t_{s}^{\prime}$ and $t_{s}$, we employ the stationary phase condition with respect to $t^{\prime}$ and $t, \partial_{t^{\prime}} \Phi_{j, j^{\prime}}=\partial_{t} \tilde{\Phi}=0$, and obtain, respectively,

$$
\begin{array}{r}
\left.\partial_{t^{\prime}} \varphi_{a_{j^{\prime}}}\left(t^{\prime}\right)\right|_{t_{s}^{\prime}}-E_{c}\left(p+A\left(t_{s}^{\prime}\right)\right)-E\left(t_{s}^{\prime}\right) x_{j^{\prime}}=0, \\
-\left.\partial_{t} \varphi_{a_{j}}(t)\right|_{t_{s}}+E_{c}\left(p+A\left(t_{s}\right)\right)+E\left(t_{s}\right) x_{j}-\omega=0 .
\end{array}
$$

Here, $\left.\partial_{t^{\prime}} \varphi_{a_{j^{\prime}}}\left(t^{\prime}\right)\right|_{t_{s}^{\prime}}$ is the time derivative of the $a_{j^{\prime}}\left(t^{\prime}\right)$ phase evaluated at the saddle-point excitation time $t_{s}^{\prime}$, $E_{c}\left(p+A\left(t_{s}^{\prime}\right)\right)$ is the conduction-band energy relation dispersion, and the term $E\left(t_{s}^{\prime}\right) x_{j^{\prime}}$ is the dipolar coupling due to the initial position $x_{j^{\prime}}$. According to Eq. (16), the phase $\varphi_{a_{j^{\prime}}}\left(t^{\prime}\right)$ is proportional to $-A\left(t^{\prime}\right) x_{q}+g_{j^{\prime}}\left(t^{\prime}\right)$. Here, $x_{q}=q a$ denotes the position of each "atom" in the lattice, and $g_{j^{\prime}}\left(t^{\prime}\right)$ is the phase related to $\tilde{\lambda}$ and $J_{q}$ functions of Eq. (16). Thus, after differentiating the phase $\varphi_{a_{j^{\prime}}}\left(t^{\prime}\right)$ with respect to time $t^{\prime}$, one can expect that the last term of Eq. (A4) cancels out in the case of $q=j_{0}=j^{\prime}$ and at $t^{\prime}=0$. This conclusion is easily obtained by considering the initial condition $a_{q}(0)=\delta_{q, j_{0}}$ of Eq. (16). However, later, at excitation times $t^{\prime}>0$, the electron should have a chance to excite from another position $j^{\prime}$, i.e., $j^{\prime}=j_{0}+1$, to the conduction band. For this scenario, the last term of Eq. (A4) does not cancel out; therefore, it contributes to the calculation of the saddle points $t_{s}^{\prime}$.

The stationary excitation times $t_{s}^{\prime}$ that will contribute most to the integral on $t^{\prime}$ of Eq. (A1) are generally complex. Then, the excitation occurs via tunneling or multiphoton processes $[27,28]$.

The structure of Eq. (A5) is similar to that of Eq. (A4). However, the difference arises with an extra term: the emitted frequency or photon energy by the solid $\omega$ and the fact that Eq. (A5) is evaluated at the recombination or emission time $t_{s}$. The energy accumulated by the electron during its propagation between $t^{\prime}$ and $t$ is emitted as a form of a photon energy $\omega$ at the recombination.

Clearly, this emitted photon energy $\omega$ is related to the phase dependence on time $\varphi_{a_{j}}(t)$, the initial and final sites $x_{j}^{\prime}$ and $x_{j}$ of the electron wave function in the lattice, and the energy dispersion relation of the conduction band $E_{c}(p+A(t))$. The phase $\varphi_{a_{j}}(t) \sim-A(t) x_{q}+g_{j}(t)$ is directly linked to the initial position $j^{\prime}$. This conclusion is easily obtained by considering the initial condition $a_{q}(0)=\delta_{q, j_{0}}$ of Eq. (16).

The saddle-point approximation on momentum $p$ can also be performed for integration of Eq. (A1). Then, to obtain the momentum $p_{s}$ that contributes to that momentum integral, the condition $\partial_{p} \Phi_{j, j^{\prime}}\left(p, t, t^{\prime}\right)=0$ reads

$$
\left.\int_{t^{\prime}}^{t} \partial_{p} E_{c}\left(p+A\left(t^{\prime \prime}\right)\right)\right|_{p_{s}} d t^{\prime \prime}+x_{j^{\prime}}-x_{j}=0 .
$$

The equation tells us that between $t^{\prime}$ and $t$ the quantity $p_{s}$ is the canonical momentum associated with the trajectory that has the dominant contribution to the harmonic emission. Note that as $\Delta x_{j^{\prime}, j}=x_{j^{\prime}}-x_{j}$ is only zero in the case of $x_{j}^{\prime}=x_{j}$, different relative excitation-recombination sites contribute to the HHG in solids. As we pointed out in the main text around Eq. (22), this can be understood as the electron being born at time $t^{\prime}$ at the position $x_{j^{\prime}}$ and recombining at time $t^{\prime}$ at any site $x_{j}$. 

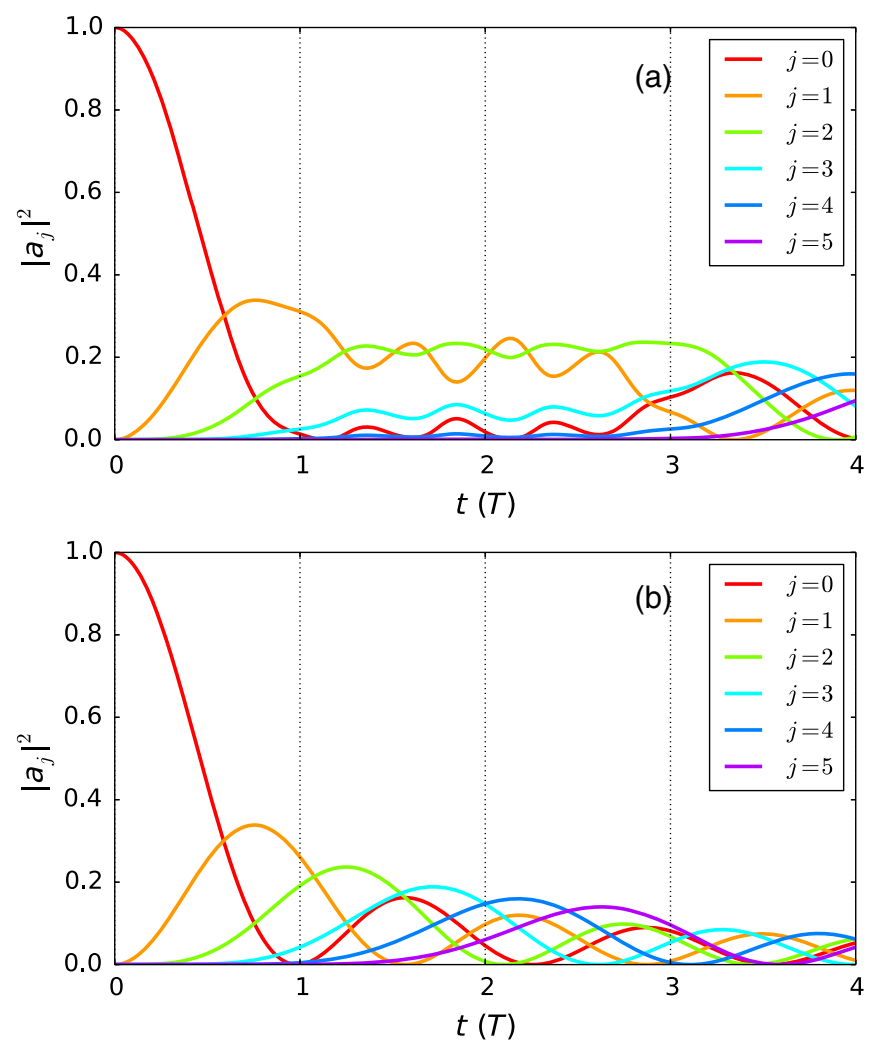

FIG. 7. (a) Evolution of the $a_{j}$ coefficients in time for $j=0,1, \ldots, 5$ for band-structure and laser-pulse parameters as in Fig. 3(a). (b) Same as (a) but with the electric field turned off $\left(\mathcal{E}_{0}=0\right)$.

\section{APPENDIX B: ELECTRON WAVE-FUNCTION SPREADING}

The coefficients $a_{j}(t)$ describe how fast the wave function $\phi_{v}(x, t)=\sum_{j} w_{v, j}(x) a_{j}(t) \quad$ spreads in the lattice. In Fig. 7(a), we plot the evolution of the $a_{j}(t)$ coefficients for $j=0,1, \ldots, 5$ for the same band-structure and laser-pulse parameters as in Fig. 3(a). The electron is initially fully localized at atom $j=0$ and gradually spreads to the first neighbors $(j=1,-1)$, the second neighbors $(j=2,-2)$, and so forth. We can see that for a narrow valence band and a short laser pulse (four periods), the electron wave function stays considerably localized throughout the whole duration of the pulse. In Fig. 7(b), we observe that turning off the electric field, $\mathcal{E}_{0}=0$, results in acceleration of the spreading of the wave function in the lattice; i.e., the subsequent atomic sites are populated faster than in the case of nonzero $\mathcal{E}_{0}$.

Note that the added probability over all the neighbors $[-j, j]$ is equal to 1 at each time. This result demonstrates that the evolution of the coefficient $a_{j}(t)$ is unitary as expected.

\section{APPENDIX C: CLASSICAL ESTIMATION OF ELECTRON TRAJECTORIES IN A 2D LATTICE}

In our paper, we state that knowledge about the localization in the HHG process is crucial, especially in the case of an elliptically polarized laser field. Therefore, to illustrate the effect of ellipticity on the HHG process, we compute the classical trajectories of an electron in an elliptical electric field. In Fig. 8, we provide an illustration of classical calculations. We consider a square lattice with a constant $a=0.52 \mathrm{~nm}$ (as used in Fig. 3), laser pulse of period $T_{0}$, and duration of $2 T_{0}$. The laser parameters are $\omega_{0}=0.0152 \mathrm{a}$.u of mean frequency (laser wavelength $\lambda_{0}=3.0 \mu \mathrm{m}$ ), and the peak intensity $I_{0}=5 \times 10^{11} \mathrm{~W} / \mathrm{cm}^{2}$. We depict the trajectories of electrons ionized from a specific lattice site (the large red dot) at the peak of the laser electric field, $0.25 T_{0}$, and close to it $\left(0.15 T_{0}, 0.2 T_{0}, 0.3 T_{0}\right.$, and $\left.0.35 T_{0}\right)$ for a few values of the ellipticity. We observe that, for high ellipticity, the excited electron travels far in the lattice. However, if the
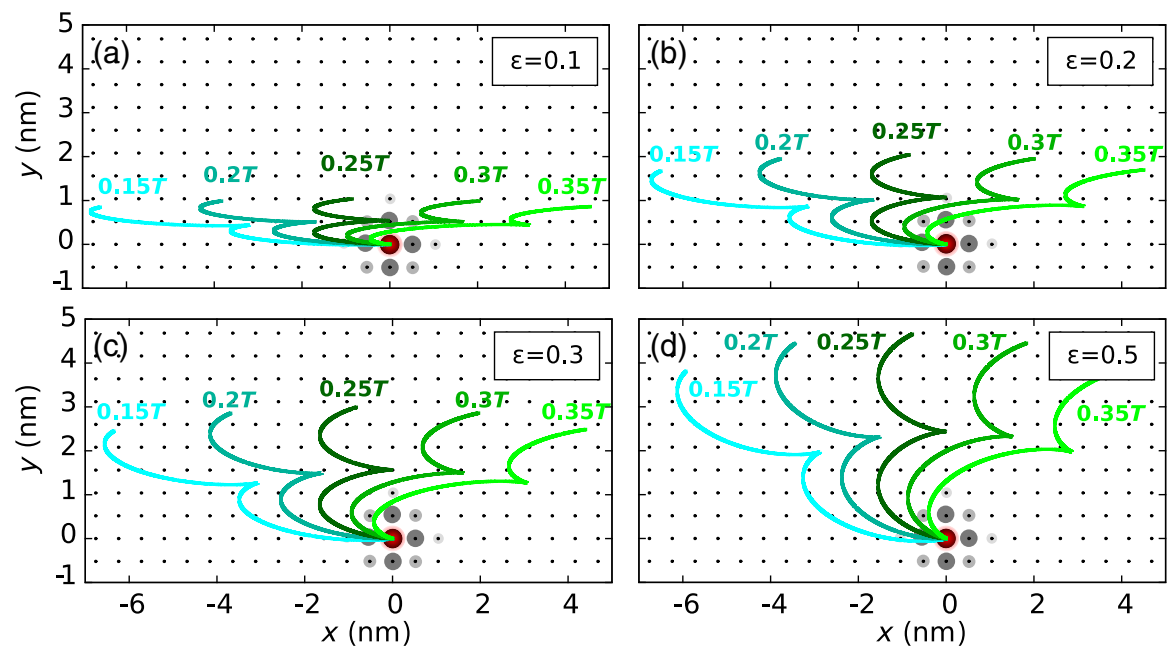

FIG. 8. Electron trajectories on a $2 \mathrm{D}$ lattice as a function of the ellipticity $\varepsilon=E_{y} / E_{x}$ calculated by solving Newton's equation of motion: (a) $\varepsilon=0.1$, (b) $\varepsilon=0.2$, (c) $\varepsilon=0.3$, and (d) $\varepsilon=0.5$. 
amplitude of the valence-band wave function is nonzero at distant neighbor sites, the recombination and HHG emission are still possible. This prevents a dramatic drop in HHG efficiency, which is observed in the atomic gas phase [33-35,35].

[1] F. Krausz and M. Ivanov, Attosecond Physics, Rev. Mod. Phys. 81, 163 (2009).

[2] M. Schultze, K. Ramasesha, C. D. Pemmaraju, S. A. Sato, D. Whitmore, A. Gandman, J. S. Prell, L. J. Borja, D. Prendergast, K. Yabana, D. M. Neumark, and S. R. Leone, Attosecond Band-Gap Dynamics in Silicon, Science 346, 1348 (2014).

[3] M. Schultze, E. M. Bothschafter, A. Sommer, S. Holzner, W. Schweinberger, M. Fiess, M. Hofstetter, R. Kienberger, V. Apalkov, V. S. Yakovlev, M. I. Stockman, and F. Krausz, Controlling Dielectrics with the Electric Field of Light, Nature 493, 75 (2013).

[4] A. Schiffrin, T. Paasch-Colberg, N. Karpowicz, V. Apalkov, D. Gerster, S Mühlbrandt, M. Korbman, J. Reichert, M. Schultze, S. Holzner et al., Optical-Field-Induced Current in Dielectrics, Nature 493, 70 (2013).

[5] S. Ghimire, A. D. DiChiara, E. Sistrunk, P. Agostini, L. F. DiMauro, and D. A. Reis, Observation of High-Order Harmonic Generation in a Bulk Crystal, Nat. Phys. 7, 138 (2011).

[6] T. T. Luu, M. Garg, S. Yu. Kruchinin, A. Moulet, M. Th. Hassan, and E. Goulielmakis, Extreme Ultraviolet HighHarmonic Spectroscopy of Solids, Nature 521, 498 (2015).

[7] H. Hohenleutner, F. Langer, O. Schubert, M. Knorr, U. Huttner, S. W. Koch, M. Kira, and R. Huber, RealTime Observation of Interfering Crystal Electrons in High-Harmonic Generation, Nature 523, 572 (2015).

[8] G. Vampa, T. J. Hammond, N. Thiré, B. E. Schmidt, F. Légaré, C. R. McDonald, T. Brabec, and P. B. Corkum, Linking High Harmonics from Gases and Solids, Nature 522, 462 (2015).

[9] G. Ndabashimiye, S. Ghimire, M. Wu, D. A. Browne, K. J. Schafer, M. B. Gaarde, and D. A. Reis, Solid-State Harmonics Beyond the Atomic Limit, Nature 534, 520 (2016).

[10] S. Neppl, R. Ernstorfer, E. M. Bothschafter, A. L. Cavalieri, D. Menzel, J. V. Barth, F. Krausz, R. Kienberger, and P. Feulner, Attosecond Time-Resolved Photoemission from Core and Valence States of Magnesium, Phys. Rev. Lett. 109, 087401 (2012).

[11] A. L. Cavalieri, N. Müller, Th. Uphues, V. S. Yakovlev, A. Baltuška, B. Horvath, B. Schmidt, L. Blümel, R. Holzwarth, S. Hendel et al., Attosecond Spectroscopy in Condensed Matter, Nature 449, 1029 (2007).

[12] S. Neppl, R. Ernstorfer, A. L. Cavalieri, C. Lemell, G. Wachter, E. Magerl, E. M. Bothschafter, M. Jobst, M. Hofstetter, U. Kleineberg et al., Direct Observation of Electron Propagation and Dielectric Screening on the Atomic Length Scale, Nature 517, 342 (2015).

[13] C. H. Zhang and U. Thumm, Streaking and Wigner Time Delays in Photoemission from Atoms and Surfaces, Phys. Rev. A 84, 033401 (2011).
[14] R. Locher, L. Castiglioni, M. Lucchini, M. Greif, L. Gallmann, J. Osterwalder, M. Hengsberger, and U. Keller, Energy-Dependent Photoemission Delays from Noble Metal Surfaces by Attosecond Interferometry, Optica 2, 405 (2015).

[15] Z. Tao, C. Chen, T. Szilvasi, M. Keller, M. Mavrikakis, H. Kapteyn, and M. Murnane, Direct Time-Domain Observation of Attosecond Final-State Lifetimes in Photoemission from Solids, Science 353, 62 (2016).

[16] O. Schubert, M. Hohenleutner, F. Langer, B. Urbanek, C. Lange, U. Huttner, D. Golde, T. Meier, M. Kira, S. W. Koch, and R. Huber, Sub-Cycle Control of Terahertz HighHarmonic Generation by Dynamical Bloch Oscillations, Nat. Photonics 8, 119 (2014).

[17] N. H. Burnett, C. Kan, and P. B. Corkum, Ellipticity and Polarization Effects in Harmonic Generation in Ionizing Neon, Phys. Rev. A 51, R3418 (1995).

[18] G. Vampa, C. R. McDonald, G. Orlando, D. D. Klug, P. B. Corkum, and T. Brabec, Theoretical Analysis of HighHarmonic Generation in Solids, Phys. Rev. Lett. 113, 073901 (2014).

[19] Y.S. You, D. A. Reis, and S. Ghimire, Anisotropic HighHarmonic Generation in Bulks Crystals, Nat. Phys. 13, 345 (2017).

[20] G. Vampa, C. R. McDonald, G. Orlando, P. B. Corkum, and T. Brabec, Semiclassical Analysis of High Harmonic Generation in Bulk Crystals, Phys. Rev. B 91, 064302 (2015).

[21] S. Ghimire, A. D. DiChiara, E. Sistrunk, G. Ndabashimiye, U. B. Szafruga, A. Mohammad, P. Agostini, L. F. DiMauro, and D. A. Reis, Generation and Propagation of High-Order Harmonics in Crystals, Phys. Rev. A 85, 043836 (2012).

[22] M. F. Ciappina et al., Attosecond Physics at the Nanoscale, Rep. Prog. Phys. 80, 054401 (2017).

[23] P. B. Corkum, Plasma Perspective on Strong Field Multiphoton Ionization, Phys. Rev. Lett. 71, 1994 (1993).

[24] A. H. Chin, O. G. Calderón, and J. Kono, Extreme Midinfrared Nonlinear Optics in Semiconductors, Phys. Rev. Lett. 86, 3292 (2001).

[25] W. Kohn, Analytic Properties of Bloch Waves and Wannier Functions, Phys. Rev. 115, 809 (1959).

[26] D. H. Dunlap and V. M. Kenkre, Dynamic Localization of a Charged Particle Moving under the Influence of an Electric Field, Phys. Rev. B 34, 3625 (1986).

[27] M. Lewenstein, Ph. Balcou, M. Yu. Ivanov, A. L'Huillier, and P. B. Corkum, Theory of High-Harmonic Generation by Low-Frequency Laser Fields, Phys. Rev. A 49, 2117 (1994).

[28] M. Y. Ivanov, M. Spanner, and O. Smirnova, Anatomy of Strong Field Ionization, J. Mod. Opt. 52, 165 (2005).

[29] C. Kittel, Introduction to Solid State Physics, 8th ed. (Wiley, Hoboken, NJ, 2005).

[30] B. E. Schmidt, A. D. Shiner, M. Giguére, Philippe Lassonde, C. A. Trallero-Herrero, J.-C. Kieffer, P. B. Corkum, D. M. Villeneuve, and F. Légaré, High Harmonic Generation with Long-Wavelength Few-Cycle Laser Pulses, J. Phys. B 45, 074008 (2012).

[31] M. Hentschel, R. Kienberger, Ch. Spielmann, G. A. Reider, N. Milosevic, T. Brabec, P. B. Corkum, U. Heinzmann, M. Drescher, and F. Krausz, Attosecond Metrology, Nature 414, 509 (2001). 
[32] G. Vampa, T. J. Hammond, N. Thiré, B. E. Schmidt, F. Légaré, C. R. McDonald, T. Brabec, D. D. Klug, and P. B. Corkum, All-Optical Reconstruction of Crystal Band Structure, Phys. Rev. Lett. 115, 193603 (2015).

[33] P. Antoine, A. LHuillier, M. Lewenstein, P. Salières, and B. Carré, Theory of High-Order Harmonic Generation by an Elliptically Polarized Laser Field, Phys. Rev. A 53, 1725 (1996).

[34] P. Dietrich, N. H. Burnett, M. Ivanov, and P. B. Corkum, High-Harmonic Generation and Correlated Two-Electron Multiphoton Ionization with Elliptically Polarized Light, Phys. Rev. A 50, R3585 (1994).
[35] A. S. Landsman, A. N. Pfeiffer, C. Hofmann, M. Smolarski, C. Cirelli, and U. Keller, Rydberg State Creation by Tunnel Ionization, New J. Phys. 15, 013001 (2013).

[36] S. Han, H. Kim, Y. W. Kim, Y.-J. Kim, S. Kim, I.-Y. Park, and S.-W. Kim, High Harmonic Generation by Field Enhanced Femtosecond Pulses in Metal-Sapphire Nanostructure, Nat. Commun. 7, 13105 (2016); G. Vampa, B. G. Ghamsari, S. S. Mousavi, T. J. Hammond, A. Olivieri, E. Lisicka-Skrek, A. Yu Naumov, D. M. Villeneuve, A. Staudte, P. Berini, and P. B. Corkum, Plasmon-Enhanced High-Harmonic Generation from Silicon, Nat. Phys., DOI: 10.1038/nphys4087 (2017). 